\title{
Molecular dynamics simulations of phase separation in the presence of surfactants
}

\author{
Laradji, Mohamed; Mouritsen, Ole G.; Toxvaerd, Søren; Zuckermann, Martin J.
}

Published in:

Physical Review E. Statistical, Nonlinear, and Soft Matter Physics

Link to article, DOI:

10.1103/PhysRevE.50.1243

Publication date:

1994

Document Version

Publisher's PDF, also known as Version of record

Link back to DTU Orbit

Citation (APA):

Laradji, M., Mouritsen, O. G., Toxvaerd, S., \& Zuckermann, M. J. (1994). Molecular dynamics simulations of phase separation in the presence of surfactants. Physical Review E. Statistical, Nonlinear, and Soft Matter Physics, 50(2), 1243-1252. https://doi.org/10.1103/PhysRevE.50.1243

\section{General rights}

Copyright and moral rights for the publications made accessible in the public portal are retained by the authors and/or other copyright owners and it is a condition of accessing publications that users recognise and abide by the legal requirements associated with these rights.

- Users may download and print one copy of any publication from the public portal for the purpose of private study or research.

- You may not further distribute the material or use it for any profit-making activity or commercial gain

- You may freely distribute the URL identifying the publication in the public portal 


\title{
Molecular dynamics simulations of phase separation in the presence of surfactants
}

\author{
Mohamed Laradji, ${ }^{1,2, *}$ Ole G. Mouritsen, ${ }^{2}$ Søren Toxvaerd ${ }^{3}$ and Martin J. Zuckermann ${ }^{1}$ \\ ${ }^{1}$ Centre for the Physics of Materials and Department of Physics, McGill University, Rutherford Building, \\ 3600, rue University, Montréal, Québec, Canada H3A 2 T8 \\ ${ }^{2}$ Department of Physical Chemistry, The Technical University of Denmark, Building 206, DK-2800 Lyngby, Denmark \\ ${ }^{3}$ Department of Chemistry, H. C. Ørsted Institute, University of Copenhagen, DK-2100 Copenhagen, Denmark
}

(Received 7 February 1994)

\begin{abstract}
The dynamics of phase separation in two-dimensional binary mixtures diluted by surfactants is studied by means of molecular dynamics simulations. In contrast to pure binary systems, characterized by an algebraic time dependence of the average domain size, we find that systems containing surfactants exhibit nonalgebraic, slow dynamics. The average domain size eventually saturates at a value inversely proportional to the surfactant concentration. We also find that phase separation in systems with different surfactant concentrations follow a crossover scaling form. Finally, although these systems do not fully phase separate, we observe a dynamical scaling which is independent of the surfactant concentration. The results of these simulations are in general in agreement with previous Langevin simulations [Laradji, Guo, Grant, and Zuckermann, J. Phys. A 44, L629 (1991)] and a theory of Ostwald ripening [Yao and Laradji, Phys. Rev. E 47, 2695 (1993)].
\end{abstract}

PACS number(s): 82.70.Kj, 64.75.+g

\section{INTRODUCTION}

The dynamics of phase separation in a binary mixture, following a thermal quench into the unstable coexistence region, proceeds by spinodal decomposition [1]. Immediately after the quench, small domains, with local concentrations roughly corresponding to that of the two immiscible phases, spontaneously form and grow and finally result in complete phase separation [1-3]. The early-time dynamics of this process is characterized by several important length scales whereas the late-time dynamics is often characterized by a single length scale corresponding, for instance, to the average size of the growing domains. This length scale is usually described by a powerlaw behavior,

$$
R(t) \sim t^{n},
$$

where the value of the growth exponent, $n$, is crucial for determining the dynamical universality class to which the system belongs, in the same manner as in critical phenomena [1]. Moreover, due to the presence of a single time-dependent length scale, the system at late times displays a self-similar behavior. Hence, dynamical scaling is usually observed in the real- and Fourier-space correlation function [1]. In this paper, we report the results of a numerical model study of the effects of surfactants on the details of the growth dynamics and the scaling in binary fluid mixtures.

During the phase-separation process, the domain growth proceeds in such a way so as to decrease the total amount of interfaces, due to the unfavorable positive

\footnotetext{
*Present address: Center for Simulational Physics, The University of Georgia, Athens, Georgia 30602.
}

coast in the interfacial energy. As a result, the driving force of the dynamics is proportional to the interfacial tension. This driving force may be diminished by adding to the mixture surfactant molecules which are surface-active particles that tend to adsorb at the interfaces which separate the growing domains [4]. This effect results from the highly anisotropic structure of surfactant molecules. A surfactant molecule usually possesses two different moieties, each having an affinity to either one of the two immiscible components. In the case of a prototype binary mixture of immiscible fluids like oil and water, a typical surfactant molecule would be an amphiphile that has a hydrophilic head preferring to be in contact with water molecules, and a hydrophobic tail preferring to be dissolved in the oil. Another typical example corresponds to $A-B$ diblock copolymers which behave as surfactants in a binary mixture of $A$ and $B$ homopolymers [5].

Under equilibrium conditions the surfactants are predominantly adsorbed at the interfaces hence effectively screening the two-body repulsive interaction between dissimilar molecules. This will result in a vanishing small value of the local interfacial tension. Similarly, we then expect that surfactants will also play an important role for the nonequilibrium behavior of these mixtures, in particular for the dynamics of the phase-separation process.

The effect of surfactants on the phase equilibria of binary mixtures such as water and oil has been extensively studied by means of experimental, theoretical, and simulational methods $[4,6,7]$. It is found that the presence of surfactants leads to the formation of a variety of microphase separated structures like microemulsions and several types of liquid-crystalline phases. The stability of these structured phases is primarily determined by the ability of the surfactant to lower the interfacial tension between the oil- and water-rich phases. 
A few studies have been reported concerning the effect of surfactants on the dynamics of phase separation. Kawakatsu and Kawasaki [8] have proposed a hybrid model, in which the two immiscible components are modeled in terms of a Ginzburg-Landau model, whereas the surfactants are treated microscopically. Laradji et al. [9] have proposed a Ginzburg-Landau model based on two local order parameters for the three components and analyzed this model by numerical Langevin simulations. More recently, Yao and Laradji [10] have analyzed surfactant-modified phase-separation dynamics using a modified Lifshitz-Slyozov theory [11]. All these studies have shown that surfactants modify the dynamics to a slow nonalgebraic growth of the domains, and that the larger the concentration of surfactants, the slower is the dynamics. Experimentally, Hashimoto and Izumitani [12] have studied the effect of adding small amounts of [poly-(styrene-block-butadiene)] diblock copolymers to a binary homopolymer mixture of polybutadiene and polystyrene and also found that polymeric surfactants slow down the phase-separation process.

An important ingredient which is, however, missing in the previous theoretical studies is the hydrodynamic forces. The reason why one should expect hydrodynamic effects to be important for phase-separation dynamics is that real systems containing surfactants most often are fluids. Moreover, the great variety of studies of phase separating fluids have very clearly shown that, in general, they exhibit different growth dynamics than binary alloys which is most likely due to the fact that fluid systems sustain hydrodynamic modes [13-25]. The presence of surfactants is not expected to suppress the hydrodynamic modes, and deviations are therefore expected from the results of the previous theoretical studies when hydrodynamic modes are properly taken into account. The purpose of the present paper is to study this issue by numerical simulation of a simple and well-defined twodimensional microscopic model using molecular dynamics methods which fully account for the effects of hydrodynamic modes. It should be emphasized that the molecular dynamics technique, in contrast to simulation techniques based on stochastic dynamics, operates on a real physical time scale.

The present numerical approach to the study of surfactant effects on phase-separation dynamics in binary systems has two major characteristics. Firstly, the quenches are performed into a fluid phase in which the hydrodynamic forces in a natural way result from the microscopic forces to which the molecules are subject. Secondly, within the model, which is built on a modification of the Lennard-Jones potential, the surfactant molecules are treated in a much more realistic way than in the previous models. This is very important if we also attempt to study phase equilibria of these systems. The simple description of surfactants employed in the Ginzburg-Landau model of Laradji et al. [9] or the modified Lifshitz-Slyozov model of Yao and Laradji [10] is not able to predict the phase behavior of mixtures of water, oil, and surfactants due to the lack of terms which account for the bending elasticity of the surfactant monolayers. Although it is not clear how the hydrodynamics modes could be included in the modified Lifshitz-Slyozov theory, it is formally possible to couple the Langevin equations in the Ginzburg-Landau model of Laradji et al. [9] to a velocity field derived from the Navier-Stokes equation, as has been done in several previous simulations of binary fluids [17-19]. However, in contrast to the description of these simple binary mixtures which is based on a single order parameter, the description of ternary systems containing surfactants has to include at least two local fields. Such a description would imply a large set of strongly coupled partial differential equations, the solution of which presents a formidable computational task.

In the present paper, the effect of surfactants on the dynamics of spinodal decomposition and phase separation in binary fluids with critical quenches is studied by means of molecular dynamics simulations for five different surfactant concentrations. The organization of the paper is as follows. In Sec. II we present the model and describe the numerical techniques used for our simulations. Section III contains three subsections: The first one deals with the time dependence of the domain growth; in the second one we discuss an ansatz for the crossover scaling behavior that describes the change in dynamics as surfactants are added to a binary fluid mixture; and the third subsection is devoted to a description of the late-time dynamical scaling behavior. Finally, the paper is concluded in Sec. IV.

\section{MODEL AND NUMERICAL METHOD}

Let us consider a two-dimensional system composed of $N_{w}$ "water" molecules, $N_{o}$ "oil" molecules, and $N_{s}$ "surfactant" molecules. For the sake of simplicity, water and oil are assumed to be represented as monatomic particles. The interaction potential is taken to be a special version of the 12-6 Lennard-Jones potential which has been used in previous calculations of phase-separation dynamics in multicomponent fluids $[20,22]$,

$$
U\left(\mathbf{r}_{i j}\right)=4 \epsilon\left[\left(\frac{\sigma}{r_{i j}}\right)^{12}-\left(2 \delta_{\alpha_{i} \alpha_{j}}-1\right)\left(\frac{\sigma}{r_{i j}}\right)^{6}\right],
$$

where $\mathbf{r}_{i j}=\mathbf{r}_{i}-\mathbf{r}_{j}$ is the distance between the two molecules $i$ and $j$. $\delta_{\alpha_{i} \alpha_{j}}$ is the Kroneker function and $\alpha_{i}$ denotes the type of molecule $i$, i.e.,

$$
\alpha_{i}= \begin{cases}1 & \text { for a water molecule } \\ 2 & \text { for an oil molecule }\end{cases}
$$

The potential in Eq. (2) ensures that molecules of different species always interact repulsively. A surfactant molecule is assumed to be a diatomic molecule with one part which is waterlike and the other part which is oillike. The two parts of the molecule are connected to each other by a harmonic spring. The intramolecular surfactant potential can therefore be written as follows:

$$
U_{s s}\left(\mathbf{r}_{i j}\right)=\frac{K_{s}}{2}\left(r_{i j}-l_{s}\right)^{2}
$$


Our choice for the harmonic potential parameters are $K_{s}=\epsilon / \sigma^{2}$ and $l_{s}=1.5 \sigma$. Since a surfactant molecule is composed of two particles (a water and an oil particle) the total number of particles in the system is $N=N_{w}+N_{o}+2 N_{s}$. In the absence of surfactants, the model exhibits both solid and fluid phases within the coexistence region. However, in this paper we shall consider only quenches into the fluid coexistence region.

The simulations are performed on two-dimensional boxes with a square geometry of linear size $L=114 \sigma$ and subject to periodic boundary conditions. The total number of particles is $N=10450$. We consider cases of symmetric mixtures (critical quenches) where the total amounts of water and oil are equal $N_{w}=N_{o}$.

Initially the system is prepared well inside the disordered one-phase region at very low average density ( $\rho=$ $N \sigma^{2} / L^{2}=0.01$ ) and then instantaneously quenched by rescaling all spatial distances in the system so that the final density is $\rho=0.8$. Immediately after the quench, the temperature is kept constant at $T=\epsilon / k_{B}$.

In our simulations, we have adopted the Nosé-Hoover technique by which one basically integrates the following Hamilton equations [26-29]:

$$
\frac{d \mathbf{r}_{i}}{d t}=\frac{\mathbf{p}_{i}}{m_{i}}
$$

and

$$
\frac{d \mathbf{p}_{i}}{d t}=\mathbf{f}_{i}-\eta \mathbf{p}_{i}
$$

where $m_{i}$ is the mass of a particle $i$ (in our case all particles have the same mass $m$ ), $\mathbf{p}_{i}$ is its momentum, and $\eta$ is a time-dependent friction parameter which varies with the total excess kinetic energy as

$$
\frac{d \eta}{d t}=\left(\sum_{i=1}^{N} \frac{\mathbf{p}_{i}^{2}}{m_{i}}-g k_{B} T\right) /\left(g k_{B} T \tau_{\eta}^{2}\right) .
$$

$g=2 N$ is the total number of degrees of freedom of the system and $\tau_{\eta}$ is a characteristic thermostat relaxation time. Equations (5) and (6) are integrated using the leap-frog algorithm,

$$
q_{i}(t+\Delta t)=q_{i}(t)+\frac{\Delta t}{m_{i}} p_{i}\left(t+\frac{\Delta t}{2}\right)
$$

and

$$
\begin{aligned}
p_{i}\left(t+\frac{\Delta t}{2}\right)= & {\left[\frac{1-\frac{\Delta t}{2} \eta(t)}{1+\frac{\Delta t}{2} \eta(t)}\right] p_{i}\left(t-\frac{\Delta t}{2}\right) } \\
& +\frac{f_{i}(t)}{m_{i}\left[1+\frac{\Delta t}{2} \eta(t)\right]}
\end{aligned}
$$

where $\Delta t$ is the time step. The algorithm, as described by Eqs. (5) and (6), is time symmetrical and thus ensures time reversibility in microscopic dynamics [29]. The integration time step is taken to be $\Delta t=0.005 \tau$, which is much smaller than the collision time [30]. We have made test runs with smaller values of the time step and found that the chosen value is sufficiently small. Five different concentrations of surfactants are considered, which
TABLE I. Characterization of the systems studied by the molecular dynamics simulations.

\begin{tabular}{cccc}
\hline \hline System & $N_{w}=N_{o}$ & \multicolumn{1}{c}{$N_{s}$} & $\rho_{s}=2 N_{s} \sigma^{2} / L^{2}$ \\
\hline System A & 4725 & 500 & $7.69 \%$ \\
System B & 4441 & 784 & $12.06 \%$ \\
System C & 4050 & 1175 & $18.08 \%$ \\
System D & 3658 & 1567 & $24.12 \%$ \\
System E & 3396 & 1829 & $28.15 \%$ \\
\hline \hline
\end{tabular}

we refer to as systems A, B, C, D, and E as described in Table I. Since the potential described in Eq. (2) is short ranged, we have truncated it at $2.5 \sigma$.

The domain growth during the phase-separation process is monitored by calculating the real-space correlation function defined as

$$
g(\mathbf{r}, t)=\langle\psi(\mathbf{r}, t) \psi(0, t)\rangle
$$

where $\psi(\mathbf{r}, t)=\left[\rho_{w}(\mathbf{r}, t)-\rho_{o}(\mathbf{r}, t)\right] / \rho$ is the local order parameter. $\rho_{w}(\mathbf{r}, t)$ and $\rho_{o}(\mathbf{r}, t)$ are the local concentrations of water and oil, respectively (including the water and the oil parts of surfactant molecules). From the correlation function, we have also calculated the structure factor which is defined as the Fourier transform of the correlation function. Assuming that the system is spatially isotropic at all times, the real and Fourier-space correlation functions are furthermore circularly averaged. The average domain size is then calculated from the first moment, $R(t)$, of the structure factor as follows:

$$
R(t)=2 \pi \frac{\sum_{q=0}^{q=q_{c}} S(q, t)}{\sum_{q=0}^{q=q_{c}} q S(q, t)},
$$

where $q_{c}$ is a large-wave-number cutoff taken to be $2 / \sigma$. This choice assures that we avoid the time-independent broad peak due to the short-range liquid ordering. We have also calculated the average domain size from the first zero of the correlation function $g(r, t)$. We shall however only present data for $R(t)$ as calculated from the structure factor, since the two length scales are found to behave in the same way.

All measured quantities are averaged over 10 independent quenches with different initial conditions, and each quench is performed over a time period of $t=2000 \tau$, where the time scale is $\tau=\sqrt{\left(m \sigma^{2} / \epsilon\right)}$.

\section{RESULTS}

\section{A. Domain growth}

Figure 1 shows snapshots of the time evolution of the patterns formed after the quench in systems B, C, and E. These snapshots show that the phase-separation process already starts at early times, and that the surfac- 
(a)
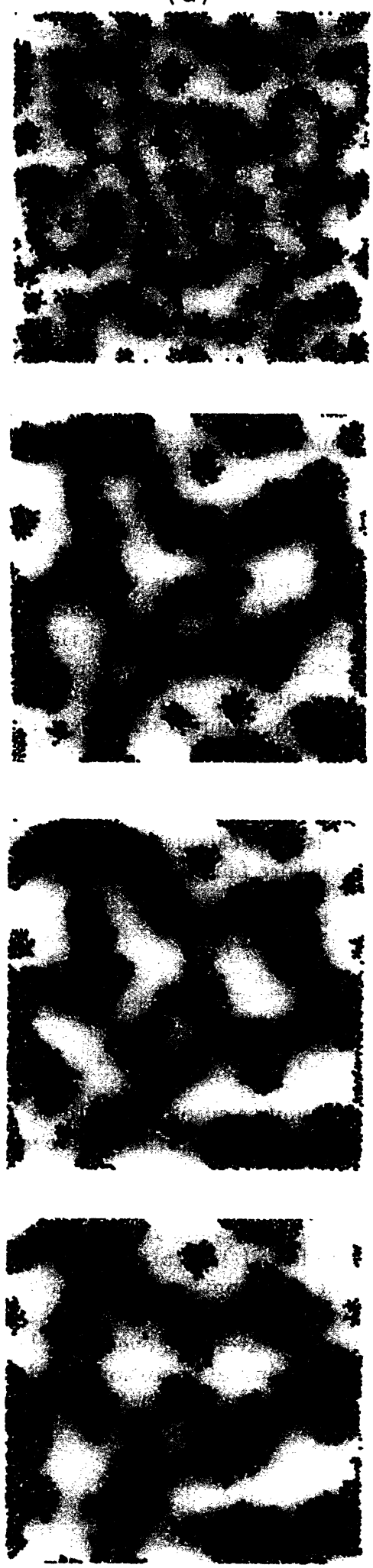

(b)
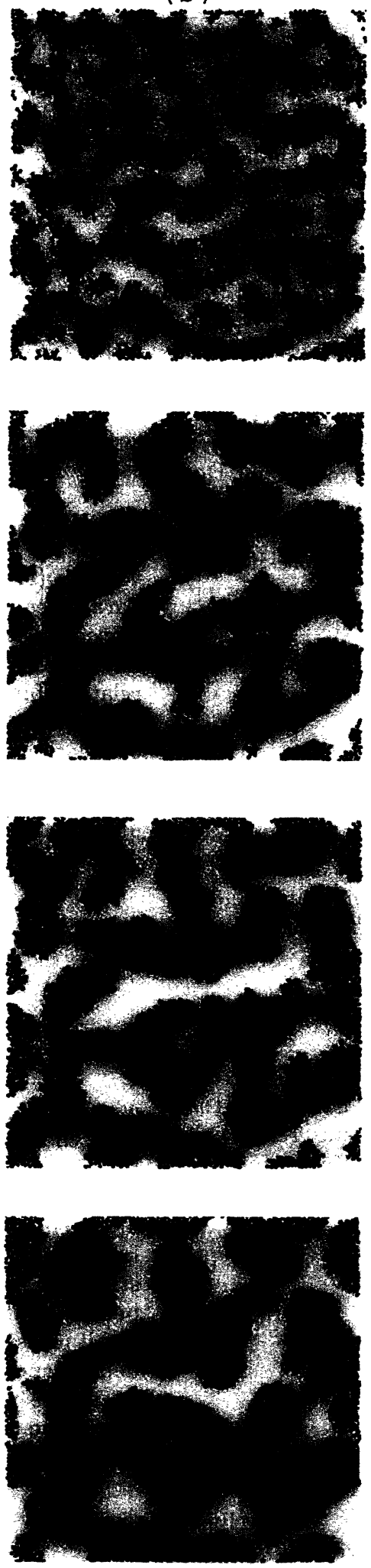
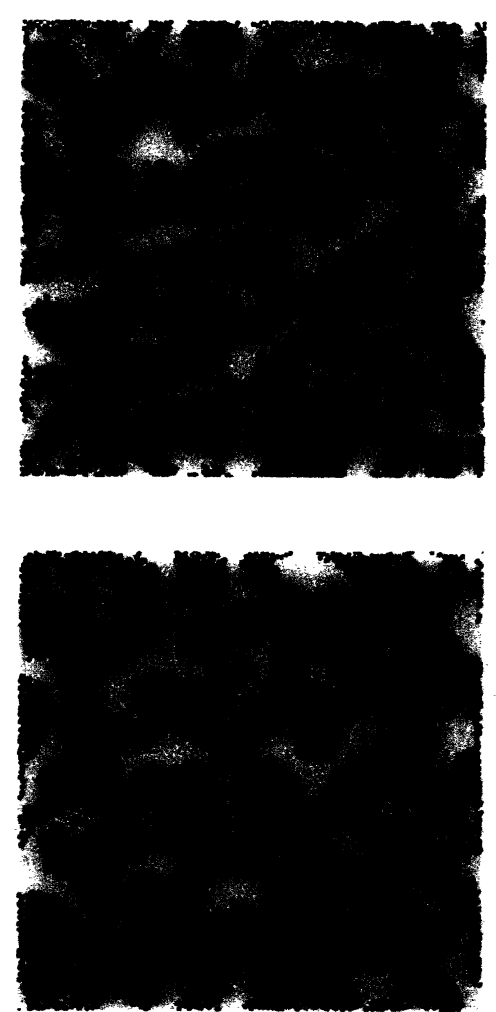

(c)
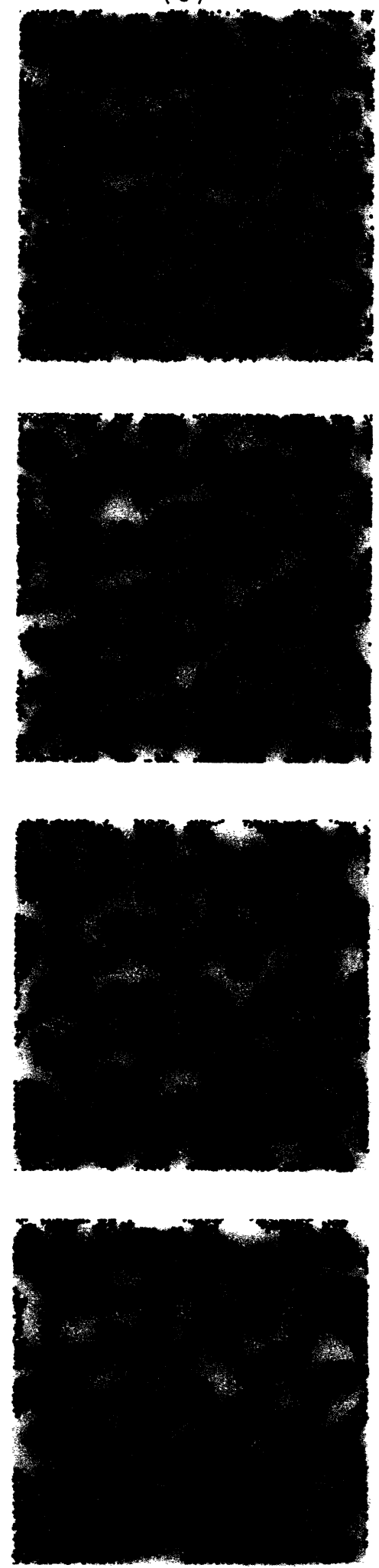

FIG. 1. Snapshots of the domain structure formed during the phase separation process for system B [column (a)], C [column (b)], and E [column (c)]. Snapshots from top to bottom correspond to times $t=100 \tau, 500 \tau, 1000 \tau$, and 2000 $\tau$, respectively. Water molecules are shown by blue dots and oil molecules by yellow dots. The hydrophilic parts of surfactants are shown by red dots and their hydrophobic counterparts by black dots. The two parts of a surfactant molecule are connected by a solid segment. 
tant molecules adsorb at the newly formed interfaces. It should be noted that if the temperature and average density parameters were chosen corresponding to a more narrow miscibility gap, the domains would have a more diffuse appearance. By comparing the snapshots of different systems at a given time, Fig. 1 shows that the average domain size is smaller for systems with higher surfactant concentrations. Hence we have the qualitative result that the growth proceeds more slowly for high surfactant concentrations. Another important observation is that the surfactant molecules tend to arrange themselves perpendicularly to the interfaces.

A quantitative measure of the average domain size calculated for all systems using Eq. (11) is displayed in Fig. 2. This figure shows that the growth initially is in a relatively fast transient regime, which is followed by a slowing down. This slowing down is clearly more pronounced the larger the surfactant concentration is.

Since the late-time growth of the average domain size is usually described by a power-law time dependence, we have in Fig. 3 redrawn in a double-logarithmic plot the time dependence of the average domain size. This figure shows very clearly that there is no surfactant concentration (even for the smallest ones as in system A) for which the growth has a power-law feature over a considerable time range. We have extracted a local growth exponent defined as $n_{\text {local }}=d \log _{10} R(t) / d t$, and found that $n_{\text {local }}$ decreases with both time and surfactant concentration, showing that the growth dynamics slows down as time evolves, as well as that this slowing down is more pronounced the larger the surfactant concentration becomes.

Based on the observation that the growth is very slow, we surmise that the final equilibrium state is rather disordered, where water and oil are segregated into small domains, which are uncorrelated at large distances, and coated by surfactant monolayers, as is the case of microemulsions $[4,6,9]$. Slowing down in domain growth has been observed in two-dimensional systems with quenched impurities $[31,32]$ or with random fields [33-35]. In these systems, the domain growth is described by a logarithmically slow activated process with $R(t) \sim(\ln t)^{\phi}$. More- over, during the time evolution of these systems, the effective surface tension diminishes with time tending towards a vanishingly small value [34]. Surfactants in a binary fluid may also be thought of as impurities, since they inhibit the growth and most probably will lead to a disordered state. However, the surfactants, unlike the impurities, are not quenched. The growth data in Figs. 2 and 3 do not conform to a logarithmic growth law. Therefore, we conclude that binary mixtures diluted by surfactants, although related to systems with quenched impurities or random fields, behave quite differently.

It is very interesting to note that, as described so far, the domain growth derived from our molecular dynamics simulations is in very good qualitative agreement with the previous observations based on the time-dependent Ginzburg-Landau model [9] and the modified LifshitzSlyozov theory [10], although as we will see later on, some details are quite different.

\section{B. Crossover scaling}

In Fig. 1 it was shown that almost all surfactants are adsorbed at the water-oil interfaces. This is because the quenches performed in our simulations are quite deep. Based on this observation, we speculate that the average domain size will eventually cease growing when all interfaces are covered by surfactants, i.e.,

$$
L(t \rightarrow \infty) \sim \rho_{s},
$$

where $L(t)$ is the total length of the interfaces per unit of area. In three dimensions, it would be the total interfacial area per unit volume. Since the average domain size, $R(t)$, is inversely proportional to $L(t)$, we therefore expect the final domain size to be inversely proportional to the average density of surfactants,

$$
R(t \rightarrow \infty) \sim \frac{1}{\rho_{s}} .
$$

Although the systems investigated did not reach the final equilibrium state within the total elapsed time of the simulations, the domain size after $2000 \tau$ seems to be

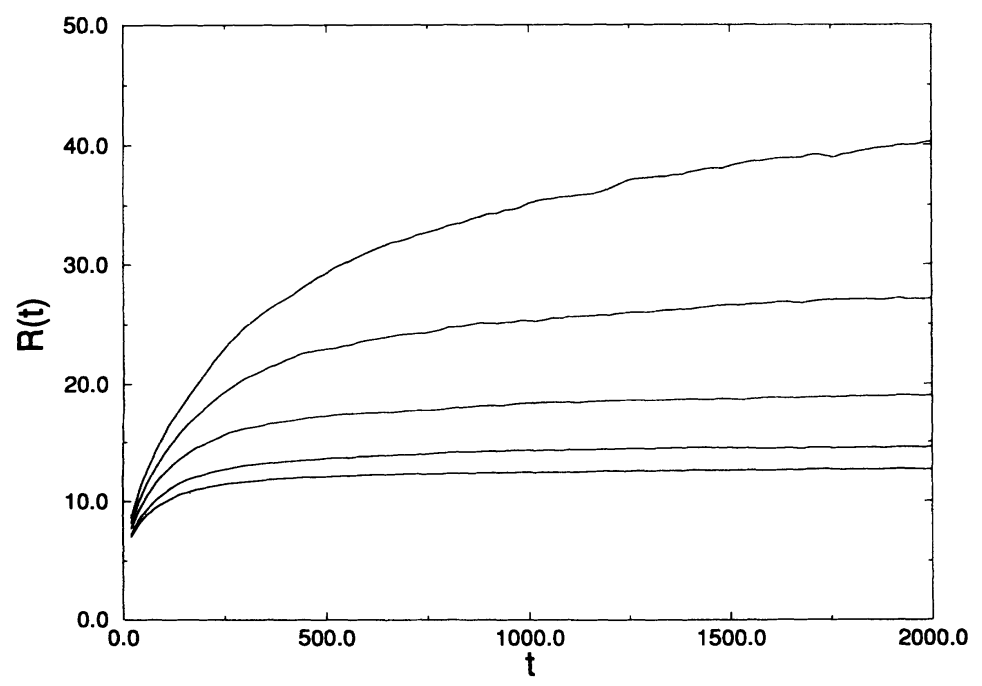

FIG. 2. Time evolution of the average domain size $R(t)$. Curves from top to bottom correspond to systems A, B, C, D, and E, respectively. $R(t)$ is in units of $\sigma$ and $t$ is in units of $\tau$. 


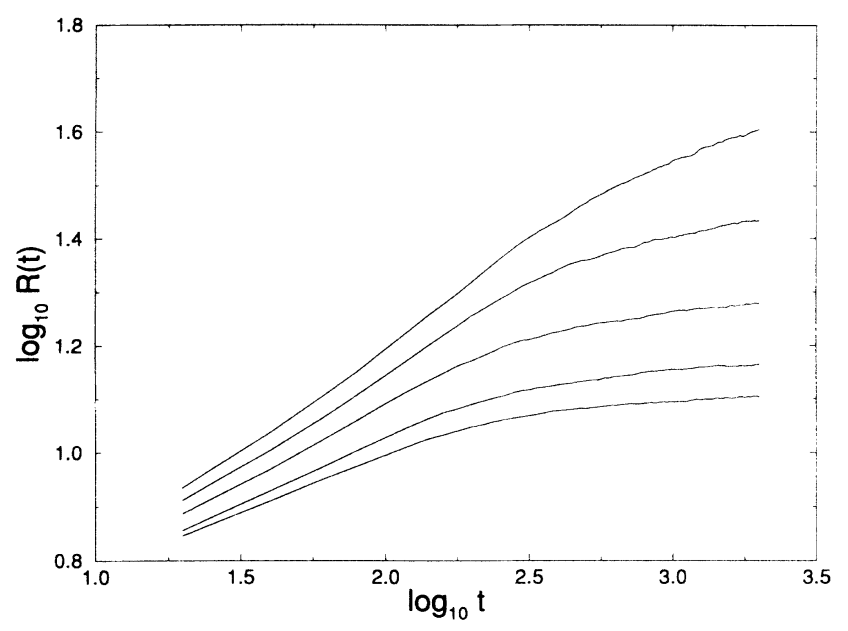

FIG. 3. Same as in Fig. 4 but in a log-log plot.

very close to saturation, especially when $\rho_{s}$ is rather large as is the case for systems D and E. Therefore in Fig. 4 we have plotted the final domain size after $t=2000 \tau$ as a function of $1 / \rho_{s}$. Assuming that systems $\mathrm{D}$ and $\mathrm{E}$ have reached saturation, we have constructed a solid line as a fit to these two points. From the figure we observe that our expectation in Eq. (13) holds rather well.

Pure binary fluids undergoing phase separation have been shown to exhibit a power-law growth behavior, where the exponent seems to depend on the spatial dimension as well as the relative composition of the two immiscible fluids. The dimensional analysis of Furukawa [25] have shown that the late-time dynamics in two-dimensional binary fluids with equal volume fractions begins with a growth exponent $n=1 / 2$ and then crosses over to a faster growth mode characterized by $n=2 / 3$. This prediction is in agreement with the recent molecular dynamics simulations of Velasco and Toxvaerd [20]. On the other hand, the dimensional analysis

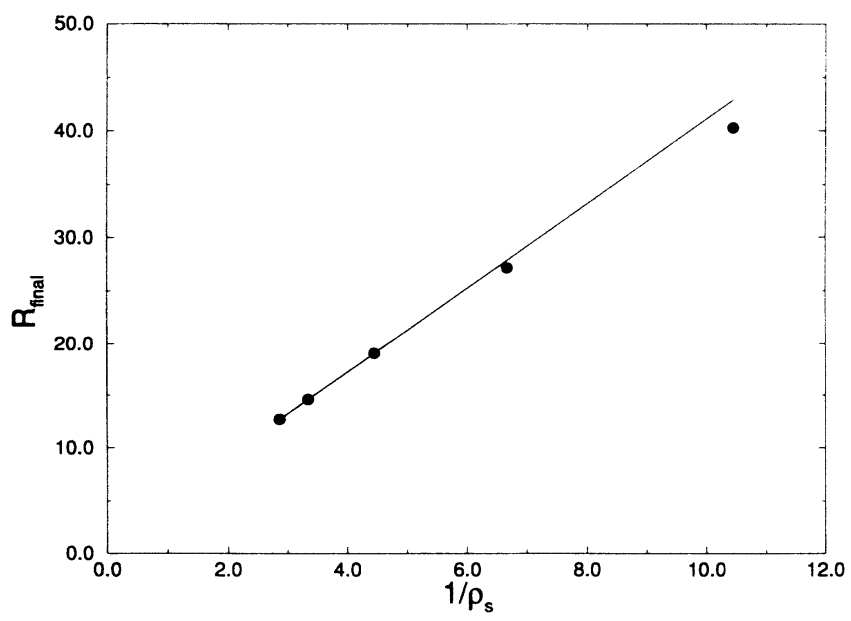

FIG. 4. Final domain size (at time $t=2000 \tau$ ) as a function of the inverse of the surfactant concentration, $1 / \rho_{s}$. The solid line is a linear fit to the two first points. of San Miguel et al. [24] predicts that the late-time dynamics follows a $t^{1 / 2}$ behavior. Other simulations using a time-dependent Ginzburg-Landau model by Farrell and Valls [16] and a lattice Boltzmann scheme by Alexander et al. [21] have shown the the dynamics at late times follows a $t^{2 / 3}$ behavior. We therefore expect a crossover scaling behavior to describe the change from the algebraic growth in pure binary fluids to a slower domain growth when surfactants are present. This crossover is described by the following ansatz:

$$
R(t)=t^{n} f\left(t \rho_{s}^{1 / n}\right)
$$

where $f(x)$ is the crossover scaling function and $x=t \rho_{s}^{1 / n}$ is the scaling variable. Notice that for large $x$, the function $f(x)$ is expected to decay as $x^{-n}$. In the previous Langevin model simulations by Laradji et al. [9] and within the modified Lifshitz-Slyozov theory by Yao and Laradji [10], this scaling ansatz was found to hold with an exponent value of $n=1 / 3$, reminiscent of the domain growth exponent in binary alloys since this previous work did not include effects caused by hydrodynamic modes.

We have tested the validity of the crossover scaling ansatz in Eq. (14) by calculating $f(x)$ for each of the five simulated systems and looking for possible data collapse. Three possible growth exponents were assumed: $n=1 / 3, n=1 / 2$, and $n=2 / 3$. When using $n=1 / 3$, no satisfactory collapse at any value of $x$ was observed. This strongly indicates that for the molecular dynamics simulations, unlike the previous Langevin simulations [9] and the modified Lifshitz-Slyozov theory [10], $n=1 / 3$ is not an appropriate exponent value. Although a fairly good data collapse for large values of $x$ was observed for $n=2 / 3$, the scaling was rather poor at small values of $x$. This might be due to the fact that $n=2 / 3$ is the growth exponent at late times in a pure binary fluid mixture as shown by the recent molecular dynamics simulations of Velasco and Toxvaerd [20]. We have then calculated $f(x)$ for $n=1 / 2$ and found that for all values of $x$, including the intermediate times, a fairly good scaling is observed as shown in Fig. 5. This crossover scaling also agrees with the recent results of Velasco and Toxvaerd [20] in the sense that the growth exponent at intermediate times is $n=1 / 2$. At large values of $x$, the crossover scaling form was found to comply with the power-law decay, but with an exponent $n=0.46$, slightly smaller than the expected one, i.e., $n=1 / 2$. The reason for this is that the function $f(x)$ decays as $x^{-n}$ only at very late times when the growth reaches saturation. As we have indicated before, our simulations however showed that none of the systems studied have fully reached saturation.

\section{Dynamical scaling}

During the late stages of phase separation in pure binary mixtures all length scales, except the microscopic ones, have the same time dependence. This leads to the following scaling property of the structure factor:

$$
S(q, t)=R(t)^{d} F(X)
$$




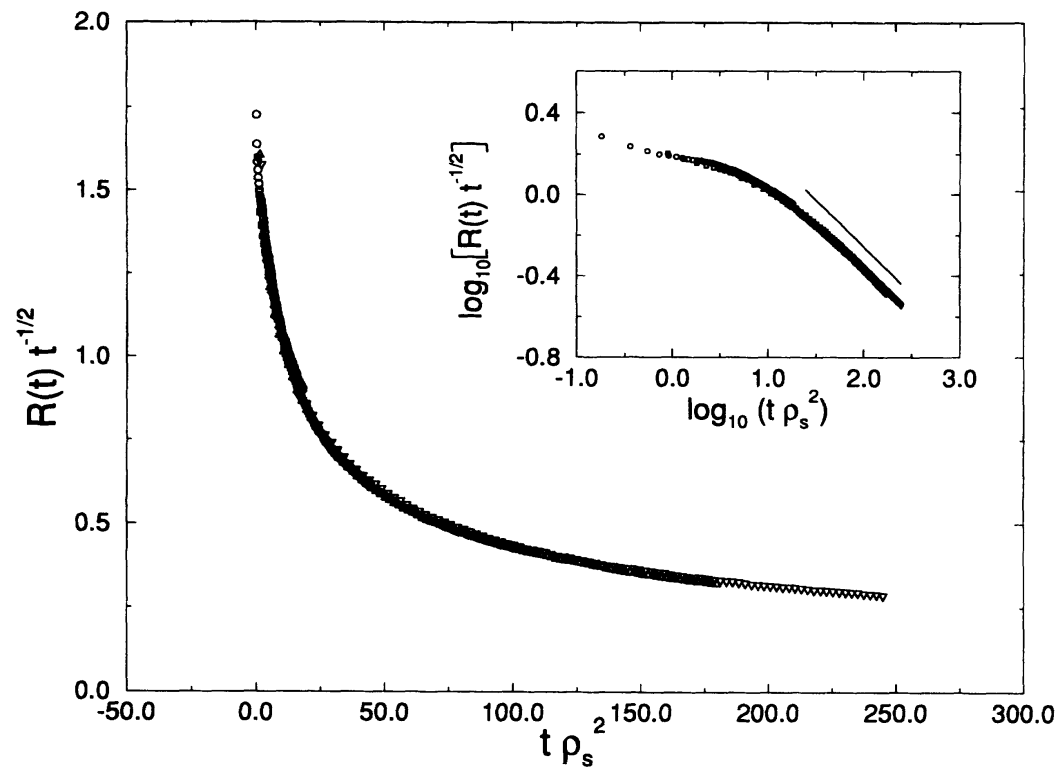

FIG. 5. Crossover scaling function defined in Eq. (14). The symbols $\circ, \square, \diamond, \triangle$, and $\nabla$ refer to systems $A$, $\mathrm{B}, \mathrm{C}, \mathrm{D}$, and $\mathrm{E}$, respectively. The inset shows the crossover scaling function in a double-logarithmic plot. The solid line has a slope of $\mathbf{- 0 . 4 6}$.

where $d$ is the spatial dimension, $X=q R(t)$ is a scaled wave vector, and $F$ is the scaling function. It should be noted that dynamical scaling is usually observed in systems which eventually undergo complete phase separation, and therefore the small length scales, which are time independent at late times, become irrelevant since their ratio to the average domain size becomes vanishingly small.

In Fig. 6, the time evolution of the structure factor for system $\mathbf{C}$ is displayed. Note that the structure factor has a well-defined peak reflecting long wavelength oscillations in the real-space correlation function. This peak indicates that domains belonging to the same species are

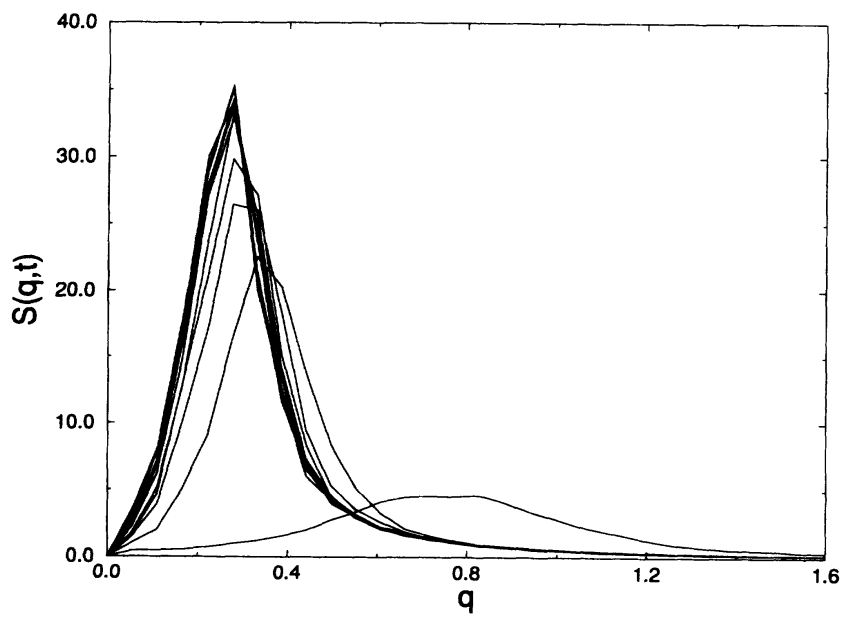

FIG. 6. Time sequence of the structure factor, $S(q, t)$, shown as a function of wave vector $q$ in system C. Structure factors from bottom to top correspond to the following times: $t=10 \tau, 200 \tau, 400 \tau, 600 \tau, 800 \tau, 1000 \tau, 1200 \tau, 1400 \tau$, $1600 \tau, 1800 \tau, 2000 \tau$. correlated within distances comparable to the average size of the domains. As time evolves, the position of the peak $q_{\max }$ decreases whereas the height of the peak increases, indicating that the domains are coarsening. As is done for pure binary mixtures, we have also examined the presence of late-time dynamical scaling in this data. In Fig. 7, the scaling function for the same system (C) is shown, and we note that dynamical scaling is observed after about $200 \tau$. It is important to point out, however, that our systems do not undergo complete phase separation, and therefore small length scales such as the interfacial width do not become infinitesimally smaller than $R(t)$. But since the domains are still much larger than

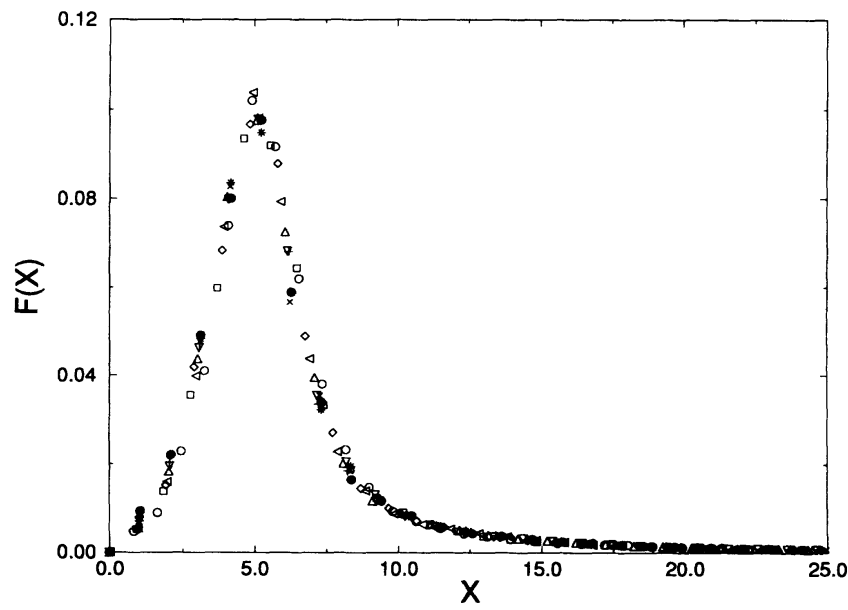

FIG. 7. Scaled structure factor, $F(X, t)=R(t)^{-2} S(q, t)$, in system $\mathrm{C}$ where $X=q R(t)$. The times shown are from $t=200 \tau$ up to $2000 \tau$ in steps of $200 \tau$ corresponding to the symbols in the sequence: $\circ, \square, \diamond, \triangleleft, \triangle, \nabla,+, \times, *, \bullet$. 
TABLE II. Exponent, $\alpha$, of Porod's law describing the tail in the structure factor, $S(q, t) \sim 1 / q^{\alpha}$, at late times.

\begin{tabular}{cc}
\hline \hline System & $\alpha$ \\
\hline System A & 2.83 \\
System B & 2.77 \\
System C & 2.68 \\
System D & 2.55 \\
System E & 2.62 \\
\hline \hline
\end{tabular}

the interfacial width, an approximate dynamical scaling is still expected to hold which is confirmed by Fig. 7 .

Scattering from thin interfaces results in a tail in the structure factor which has the form

$$
S(q) \sim \frac{1}{q^{\alpha}}
$$

This is known as Porod's law. For flat and thin interfaces, the exponent in Porod's law is $\alpha=d+1$. However, a roughening of the interfaces leads to a reduction in the exponent value. The values obtained for $\alpha$ for the present set of data are shown in Table II. This table shows that as the surfactant concentration is increased, the exponent in Porod's law becomes smaller, most probably due to an increased roughening of the interfaces as $\rho_{s}$ increases. However, the exponent values are still quite close to the predicted ones for thin interfaces.

Finally, we have observed that the scaling function for the structure factor is independent of the surfactant concentration, $\rho_{s}$, as demonstrated in Fig. 8. This is a very interesting result which suggests that the structure of a system with a large value of $\rho_{s}$ at a given late time looks basically the same as that of a system with a smaller value of $\rho_{s}$ at an earlier time. It should be noted, however, that the scaling function for system A does not comply with the other ones. We believe that this discrepancy is due to the finite-size effects encountered in system $\mathrm{A}$ at late times, since in this system the domains become quite large.

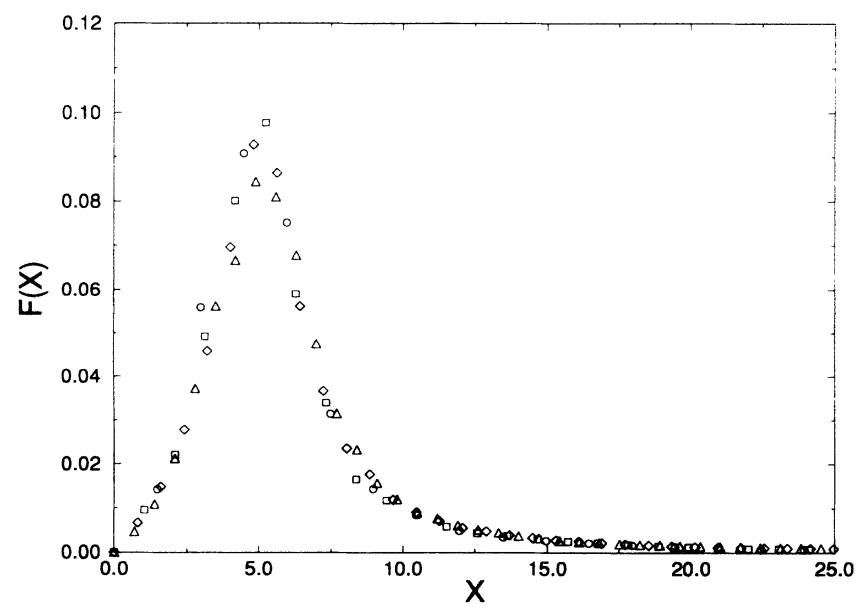

FIG. 8. Scaled structure factor for systems B (o), C ( $\square)$, $\mathrm{D}(\triangle)$, and $\mathrm{E}(\diamond)$.

\section{SUMMARY AND DISCUSSION}

By using molecular dynamics simulations and a simple interaction potential of a two-dimensional model, we have studied the dynamics of phase separation in binary fluids, such as mixtures of water and oil, in the presence of surfactants, such as amphiphiles. Despite the complicated molecular structure of the molecules involved in the real systems, we have chosen to simulate the molecules of water and oil by simple monatomic particles, and to treat a surfactant as a diatomic molecule, with one part being waterlike and the other part being oil-like. For further simplification, we have made the model potential completely symmetric under the exchange between water and oil molecules.

The dynamics of phase separation in these systems has previously been studied by a simulation of a timedependent Ginzburg-Landau model [9] and by a modified Lifshitz-Slyozov theory [10]. Unlike these studies, the approach in this paper was to use a model in which surfactants are treated more realistically. Moreover, the dynamics of the model was examined by a real-time molecular dynamics technique that takes into account the hydrodynamic modes in a natural manner which is important since the quenches were performed into the fluid coexistence region of the pure binary mixture. This is very important since many previous studies have shown that binary fluids, if quenched into their coexistence region, evolve towards equilibrium via a dynamics which most probably belong to a different dynamical universality class than alloys $[23,25,24,20]$.

The main result of the present paper is that the growth dynamics in binary fluid mixtures is drastically affected by surfactants. In fact, a nonzero surfactant concentration leads at late times to a dynamics which is slow and nonalgebraic. Moreover, the larger the surfactant concentration, the slower is the dynamics. The occurrence of this behavior results from the molecular structure of surfactants which favor interfacial adsorption, which leads to an effective screening of the two-body repulsive interaction between water and oil molecules. This reduces the effective interfacial tension. The reduction in interfacial tension is time dependent since the local interfacial surfactant concentration increases as time evolves. The domain growth eventually stops when all interfaces are covered by surfactants. These findings are qualitatively in agreement with the previous time-dependent GinzburgLandau simulations [9] and the analytical results of the modified Lifshitz-Slyozov theory [10]. A model related to ours has been used by Smit and co-workers [36] to calculate the surface tension of a water-oil interface when occupied by surfactants from which it was found that the surface tension reduces linearly with increasing surfactant concentration. It should be noted that the recent experiment by Hashimoto and Izumitani [12] on the effect of small amounts of diblock copolymers on the dynamics of binary homopolymers is also in qualitative agreement with the present simulations.

Another interesting result of our work is that the final average domain size is found to scale as $1 / \rho_{s}$, also in agreement with the time-dependent Ginzburg-Landau 
simulations and the modified Lifshitz-Slyozov theory. This leads us to observe a crossover scaling form which unifies the growth dynamics in pure binary fluids with the phase-separation dynamics in systems which contain surfactants. Unlike the previous studies, however, the crossover-scaling function found here reflects the importance of hydrodynamic modes, since the crossover exponent was found to be $n=1 / 2$ instead of $n=1 / 3$, which was observed in the previous studies $[9,10]$.

Finally, as in pure binary fluids, a self-similar behavior was observed at late times, here demonstrated via the dynamical scaling property of the structure factor. Furthermore, the associated scaling functions were found to be invariant under changes of the surfactant concentration.

Although the model presented in Sec. II was here used only to study the nonequilibrium behavior of water-oilsurfactant mixtures, it should also be of great interest in a study of the equilibrium properties of these systems. Although it is found that the domain size saturates at very late times, we are unable from this finite-time finitesystem observation to make definite conclusions regarding the nature of equilibrium phase of the ternary mixture. It is possible that our systems will equilibrate into a microemulsion structure. However, for the structure factor found in our simulation, although it has a distinct peak, intensity tends to zero as $q$ goes to zero, whereas in a proper microemulsion phase it will tend towards a finite value. It is also possible that the final states will have a lamellar structure. We assess that the time required to obtain the final equilibrium structures is likely to be orders of magnitude longer than the total elapsed time in our simulations. Hence other numerical methods should be used in order to elucidate the final equilibrium state.

The present model simulations were performed in two spatial dimensions, whereas the physical systems in question are three dimensional. However, due to our current computer time and memory limitations, only systems with a rather small number of molecules can be treated in three dimensions and an insufficient number of independent runs can be performed. Therefore, we are not able at present to generate reliable data for phase-separation dynamics in three dimensions using the present model approach. Although the general features of the dynamics with a crossover scaling and a self-similar behavior are certainly expected to hold in three as well as in two dimensions, details like the value of the exponent describing the crossover scaling function are expected to depend on dimension, in particular because the dynamics of a pure binary fluid for critical quenches in three dimensions is different from that in two dimensions.

\section{ACKNOWLEDGMENTS}

This work was supported by the Danish Natural Research Council under Grant No. 11-0065, by the Natural Sciences and Engineering Research Council of Canada, and les Fonds pour la Formation des Chercheurs et l'Aide à la Recherche de la Province de Québec via both a center and a team grant. The stay of M.L. in Denmark was supported by the Danish Natural Science Research Council under Grant No. 11-0474-1.
[1] J.D. Gunton, M. San Miguel, and P.S. Sahni, in Phase Transitions and Critical Phenomena, edited by C. Domb and J.L. Lebowitz (Academic Press, New York, 1983), Vol. 8, p. 265.

[2] Dynamics of Ordering Processes in Condensed Matter, edited by S. Komura and H. Furukawa (Plenum Press, New York, 1987), p. 574.

[3] O.G. Mouritsen, in Kinetics of Ordering and Growth at Surfaces, edited by M.G. Lagally (Plenum, New York, 1990), p. 1.

[4] Physics of Amphiphilic Layers, edited by J. Meunier, D. Langevin, and N. Boccara (Springer-Verlag, Berlin, 1987).

[5] R. Holyst and M. Schick, J. Chem. Phys. 96, 7728 (1992).

[6] G. Gompper and M. Schick, Phys. Rev. Lett. 62, 1647 (1989); Phys. Rev. A 42, 2137 (1990); Phys. Rev. B 41, 9148 (1991).

[7] M. Laradji, H. Guo, M. Grant, and M.J. Zuckermann, Phys. Rev. A 44, 8148 (1991).

[8] K. Kawasaki and T. Kawakatsu, Physica 164, 549 (1990); T. Kawakatsu and K. Kawasaki, ibid. 167, 690 (1990).

[9] M. Laradji, H. Guo, M. Grant, and M.J. Zuckermann, J. Phys. A: Math. Gen. 24, L629 (1991); J. Phys. Condens. Matter 4, 6715 (1992).

[10] J.H. Yao and M. Laradji, Phys. Rev. E 47, 2695 (1993).

[11] I.L. Lifshitz and V.V. Slyozov, J. Phys. Chem. Solids 19, 35 (1962).
[12] T. Hashimoto and T. Izumitani, Macromolecules 26, 3631 (1993).

[13] N.-C. Wong and C. Knobler, Phys. Rev. A 24, 3205 (1981).

[14] P. Guenoun, R. Gastaud, F. Perrot, and D. Beysens, Phys. Rev. A 36, 4876 (1987).

[15] T. Izumitani, M. Takenak, and T. Hashimoto, J. Chem. Phys. 92, 3213 (1990).

[16] J.E. Farrell and O.T. Valls, Phys. Rev. B 40, 7027 (1989).

[17] T. Koga and K. Kawasaki, Phys. Rev. A 44, R817 (1991).

[18] S. Puri and B. Dunweg, Phys. Rev. A 45, R6977 (1992).

[19] O.T. Valls and J. Farell, Phys. Rev. E 47, R36 (1993).

[20] E. Velasco and S. Toxvaerd, Phys. Rev. Lett. 71, 388 (1993).

[21] F.J. Alexander, S. Chen, and D.W. Grunau, Phys. Rev. B 48, 634 (1993).

[22] M. Laradji, O.G. Mouritsen, and S. Toxvaerd (unpublished).

[23] E.D. Siggia, Phys. Rev. A 20, 595 (1979).

[24] M. San Miguel, M. Grant, and J.D. Gunton, Phys. Rev. A 31, 1001 (1985).

[25] H. Furukawa, Adv. Phys. 34, 703 (1985).

[26] S. Nosé, Mol. Phys. 52, 255 (1984).

[27] W.G. Hoover, Phys. Rev. A 31, 1695 (1985).

[28] S. Toxvaerd, Mol. Phys. 72, 159 (1991).

[29] S. Toxvaerd, Phys. Rev. E 47, 343 (1993).

[30] The mean collision time can be calculated from the mem- 
ory function and the appropriate Mori coefficients and a. value of $0.05 \tau$ is found for the two-dimensional LennardJones system at $T=\epsilon / k_{B}$ and $\rho \sigma^{2}=0.8$ [S. Toxvaerd, J. Chem. Phys. 81, 5131 (1984)].

[31] G.S. Grest and D.J. Srolovitz, Phys. Rev. B 32, 3014 (1985); D.J. Srolovitz and G.S. Grest, ibid. 32, 3021 (1985).

[32] D. Chowdhury, M. Grant, and J.D. Gunton, Phys. Rev. B 35, 6792 (1987).
[33] J. Villain, Phys. Rev. Lett. 52, 1543 (1984).

[34] M. Grant and J.D. Gunton, Phys. Rev. B 35, 4922 (1987).

[35] E. Orguz, A. Chakrabarti, R. Toral, and J.D. Gunton, Phys. Rev. B 42, 704 (1990).

[36] B. Smit, A.J. Hilbert, K. Esselink, L.A.M. Rupert, N.M. van Os, and A.G. Schilijper, J. Chem. Phys. 95, 6362 (1991). 
(a)
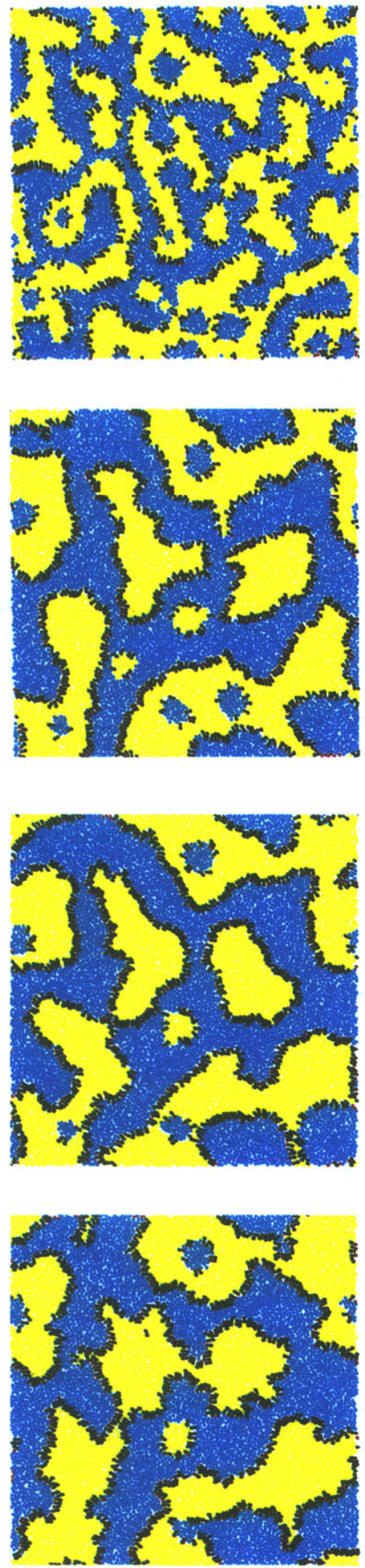

(b)
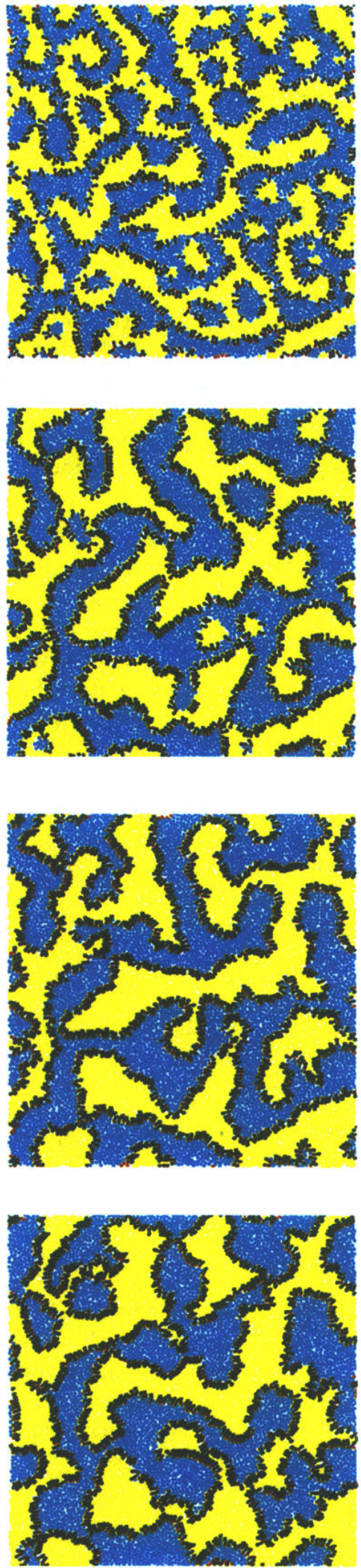

(c)
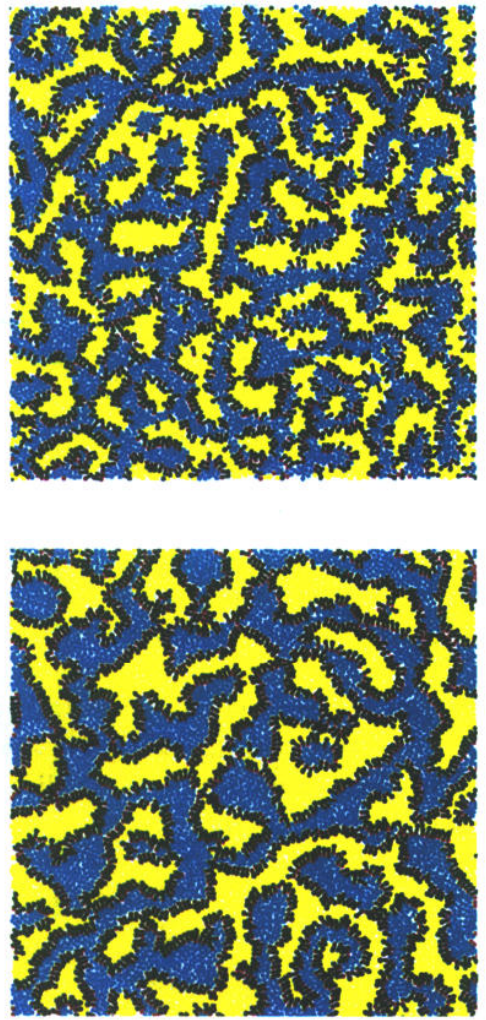

FIG. 1. Snapshots of the domain structure formed during the phase separation process for system B [column (a)], C [column (b)], and E [column (c)]. Snapshots from top to bottom correspond to times $t=100 \tau, 500 \tau, 1000 \tau$, and $2000 \tau$, respectively. Water molecules are shown by blue dots and oil molecules by yellow dots. The hydrophilic parts of surfactants are shown by red dots and their hydrophobic counterparts by black dots. The two parts of a surfactant molecule are connected by a solid segment. 